\title{
Scrutinization of FOCDMA Code families for Application in Optical Communication
}

\author{
C.S.Mishra*1, M.Ravikumar ${ }^{2}$ \\ ${ }^{1}$ Gandhi Institute Technological Advancement (GITA) Bhubaneswar \\ ${ }^{2}$ Malaviya National Institute of Technology, Jaipur
}

\section{Article Info \\ Article history: \\ Received Jan 18, 2017 \\ Revised Feb 28, 2017 \\ Accepted Mar 20, 2017}

\section{Keywords:}

FOCDMA

MAI

Orthogonal codes

\begin{abstract}
In code division multiple access (CDMA) code construction and analysis, the bit error rate due to multiple access interference is an important performance parameter which is overcome to some extent by generating almost orthogonal codes. Ideal orthogonal code families should have zero autocorrelation and no crosscorrelation. The building of fiber-optic CDMA (FOCDMA) is based on binary, unipolar spreading codes, which in turn requires considerably longer length of spreading codes in order to satisfy these constraints. Spreading an optical bit in wavelength, time and multiple fibers is observed to satisfy the constraints for accommodating sufficiently large number of users with a comparatively smaller spreading code length. Various optical code families of different dimensions have been proposed for FOCDMA. The performance of the code families varies under different conditions. In this research, some aspects of the performance issues have been considered.
\end{abstract}

Copyright (c) 2017 Institute of Advanced Engineering and Science. All rights reserved.

\section{Corresponding Author:}

C.S.Mishr,

Gandhi Institute Technological Advancement (GITA) Bhubaneswar, India

Email: csmisra2010@gmail.com

\section{INTRODUCTION}

Now a day's communication network plays on important issue for the sake of development of society, security and economy. The improvement of communication network is required in the world particularly the optical communication network. The concept of FOCDMA is based on widely used code division multiple access (CDMA)[1] technology in microwave wireless communications, where users are assigned signature sequences or spreading codes. A common optical communication channel (FOCDMA), which shared among multiple users. The bit error rate (BER) is the most significant routine parameter of any digital optical communications system. The probability of error is that any given bit will have been received from transmitter to receiver in the communication channel. For example a standard maximum bit error rate specified for many systems is $10-9[6,7,8]$. This indicates that the receiver is authorized to produce a maximum of 1 error in every 109 bits of information transmitted or, putting it another way, the probability that any received bit 10-9 in an error. The primarily BER(bit error rate) depends on the signal to noise ratio (SNR) of the reached signal which is evaluated by the transmitted signal power, the attenuation of the connection, the link scattering and the receiver noise [9-12]. Measurement of the BER is not a trivial process and needs sophisticated and high-priced equipment to attain correctness, principally at high bit rates. on the other hand, the special effects of noise and other signal degradation processes can be investigated properly and possibly even in a pseudo quantitative and BER degradation trends can be enthusiastically found by reason of the effects of signal attenuation and dispersion [13-16].

This paper shows a comparative study of performance analysis for different sets of parameters. The different parameters considered are cardinality, spreading factor, number of wavelengths, temporal length and weight. The performance criterion of these code families is bit error rate (BER) due to MAI as a function 
of number of active users [17-20]. The effect of one parameter on other using four types of codes is analysed and discussed. The two-dimensional code families considered in this comparison are GMWRSC, RWOP, CRWOP and MWOOC [21-23]. The comparisons are shown in the form of tabulations and figures. In tabulations, each row contains a parameter, i.e., cardinality (Nmax), BER due to MAI, spreading factor (Sf), weight (K'), number of wavelengths (Wl) and number of time chips (T). In the tabulation, each column represents the parameters corresponding to each of the four code families [24,25]. The objective is to find optimum code family with which maximum number of users should use the spectrum with less BER due to MAI. As number of users increases BER also increases. The code families which show less increase in BER are better. For the sake of same comparison, we use generalised BER form, which given by [2-5]

$$
\begin{aligned}
\mathrm{BER}^{\prime} & =\frac{1}{2} \sum_{\mathrm{i}=\mathrm{K}^{\prime}}^{\mathrm{N}} \mathrm{N}_{\mathrm{C}_{\mathrm{i}}}\left(\frac{\mathrm{K}^{\prime 2}}{2 \mathrm{WT}}\right)^{\mathrm{i}}\left(1-\frac{\mathrm{K}^{2}}{2 \mathrm{WT}}\right)^{\mathrm{N}-\mathrm{i}} \\
\mathrm{BER}^{\prime} & =\frac{1}{2} \sum_{\mathrm{i}=0}^{\mathrm{K}^{\prime}}(-1)^{\mathrm{i}} \mathrm{K}_{\mathrm{C}_{\mathrm{i}}}\left(1-\frac{\mathrm{qi}}{\mathrm{K}^{\prime}}\right)^{\mathrm{N}-1}
\end{aligned}
$$

Equation 1(1) and (2) represent the bit error rate (BER) for gmwrcs and mwoocs respectively

The BER (bit error rate) owing to MAI with respect to to wavelengths wi and wj are represented as BER (Nwi) and BER (Nwj). Since the two 1D OOCs along wavelengths wi and wj are detected simultaneously for any user, an error in detection is possible provided that overlaps from the interfere users on wavelengths wi and wj are bit synchronous (T), so the bit error rate due to MAI for the 2D code families is

$$
\operatorname{BER}^{\prime}=\frac{\operatorname{BER}\left(\mathrm{N}_{\mathrm{wi}}\right) \times \mathrm{BER}\left(\mathrm{N}_{\mathrm{wj}}\right)}{\mathrm{T}}
$$

Equation (3) represents the bit error rate (BER) for CRWOP and RWOP.

This research paper examined the performance of fiber optic code division multiple access (FOCDMA) code families in the course of different set of parameters. The different code families are realized by generalized multi-wavelength Reed-Solomon code (GMWRSC), row-wise orthogonal pairs(RWOP), complete row-wise orthogonal pairs (CRWOP), Multiwavelength OOC (MWOOC), where different sets of parameters are realized by number of maximum users (Nmax) Bit error rate (BER), Spreading Factor (Sf), Weight (K'), Number of Wavelengths(WI), and number of time chips (T).

\section{RESULT AND DISCUSSION}

To investigate the performance of different code family, different sorts of parameters such as cardinality, Spreading factor, number of wave lengths, temporal chips and weights are considered. Bit error rate (BER) is an important criterion of code family which leads efficiency of optical fiber. To obtain BER, The two dimensional code families, such as GMWRSC, RWOP, CRWOP and MWOOP are compared. These code families are scrutinized by the variation of different parameters. The objective of this study is to find optimum code family with which maximum number users should use the spectrum with less bit error rate. This analysis is made as follows.

\subsection{Analysis of code family based on cardinality}

The parameters, cardinality or number of maximum parameter (Nmax) plays an important to discuss the efficiency of code family. We have chosen number of users Nmax 169 for GMWRSC and C RWOP, 168 RWOP and 162 for MWOOC. Using above Nmax values in their respective code families, different parameters such as BER, Sf, K', WI and T are generated which shown in Table 1.

Table 1. Variation of BER with similar cardinality for four types of code families

\begin{tabular}{lllll}
\hline & GMWRSC & CRWOP & RWOP & MWOOC \\
\hline Nmax & 169 & 169 & 168 & 162 \\
BER & .1735 & .0003036 & .0001609 & .003443 \\
Sf & 2028 & 702 & 780 & 1623 \\
K' & 12 & 4 & 4 & 6 \\
Wl & 13 & 26 & 20 & 3 \\
T & 156 & 27 & 39 & 541 \\
\hline
\end{tabular}




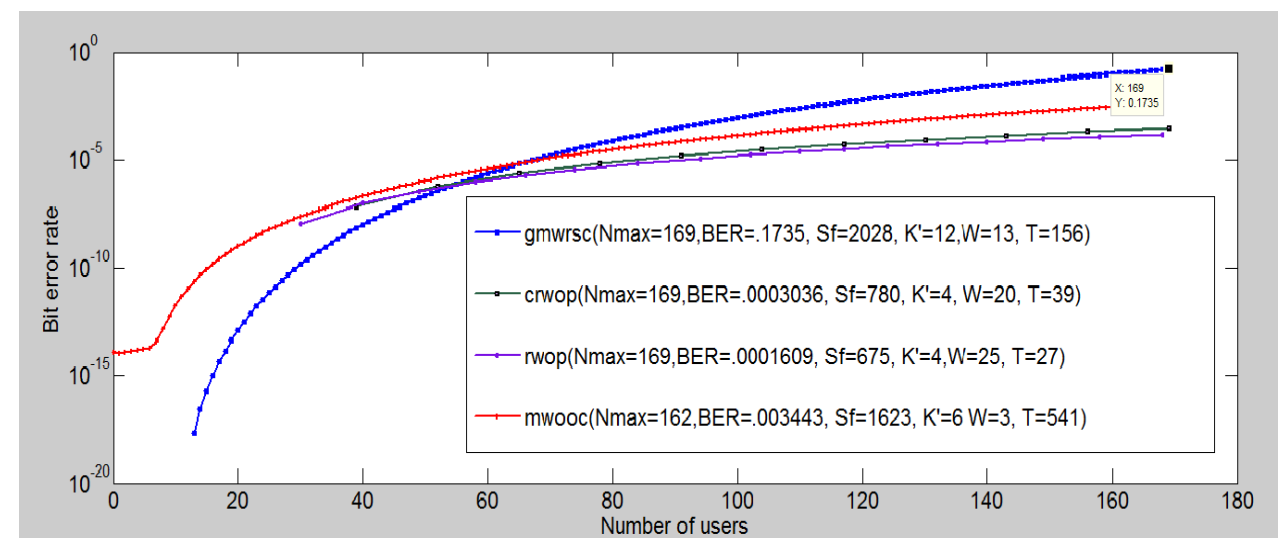

Figure 1. BER vs Number of users with similar cardinality

Using data from Table1, a graph is plotted for the sake of comparison of different code family, which is shown in Figure 1. Figure 1 gives information about the variation of bit error rate with respect to number of users (x-axis) where parameters which are generated from different code family and also mentioned in this figure. According data Table 1 and analysing Figure 1, it is inferred that RWOP gives better efficiency then other. Similarly using same principle, cardinality also increased up to 375 such as 361 for GMWRSC and CROWP, 385 for RWOP and 375 for MWOOC. Corresponding each Nmax, different parameters like Table 1 is also suitably generated for their code family, which is shown Table 2.

Table 2. Variation of BER with similar cardinality for four types of code families

\begin{tabular}{lllll}
\hline & GMWRSC & CRWOP & RWOP & MWOOC \\
\hline Nmax & 361 & 361 & 385 & 375 \\
BER & .01483 & .0002595 & .0001257 & .02321 \\
Sf & 6498 & 1482 & 1770 & 2255 \\
K $^{\prime}$ & 18 & 4 & 4 & 6 \\
WI & 19 & 38 & 30 & 5 \\
T & 342 & 39 & 59 & 451 \\
\hline
\end{tabular}

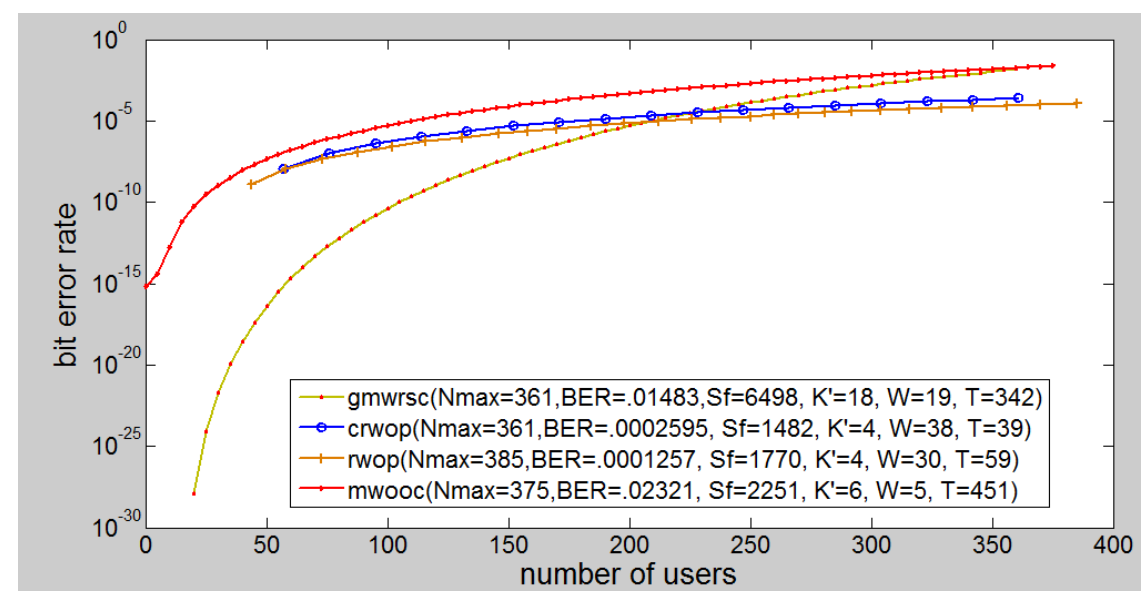

Figure 2. BER vs Number of users with similar cardinality

Using data from Table 2 again a graph is plotted between BER (y-axis) with respect to number of users (along $\mathrm{X}$-axis) for different code family. It is also revealed from Table 2 and Figure 2 that RWOP gives less bit error as compared other code family. 


\subsection{Analysis code family based on Equivalent Spreading Factor}

Like cardinality, spreading factor is also a key parameter to minimize the BER. So spreading factor in different code families generated different type of parameters such as BER, Nmax, WI, and T. Here spreading factor is taken 714 for GMWRSC, 702 for CRWOP, 780 for RWOP, 723 for MWOOC. Using these values the above mentioned parameters are generated with respect to their code family. These parameters with respect to different code families are suitable mentioned in Table 3. Using data from Table 3 , a graph is plotted between numbers of users ( $\mathrm{x}$-axis) Bit error rate (y-axis), which is shown in Figure 3.

Table 2.3 Variation of BER with similar spreading factor for four types of code families

\begin{tabular}{lllll}
\hline & GMWRSC & CRWOP & RWOP & MWOOC \\
\hline Sf & 714 & 702 & 780 & 723 \\
BER & .9307 & .0003036 & .0001609 & .001292 \\
Nmax & 119 & 169 & 168 & 72 \\
K' $^{\prime}$ & 6 & 4 & 4 & 6 \\
WI & 7 & 26 & 20 & 3 \\
T & 102 & 27 & 39 & 241 \\
\hline
\end{tabular}

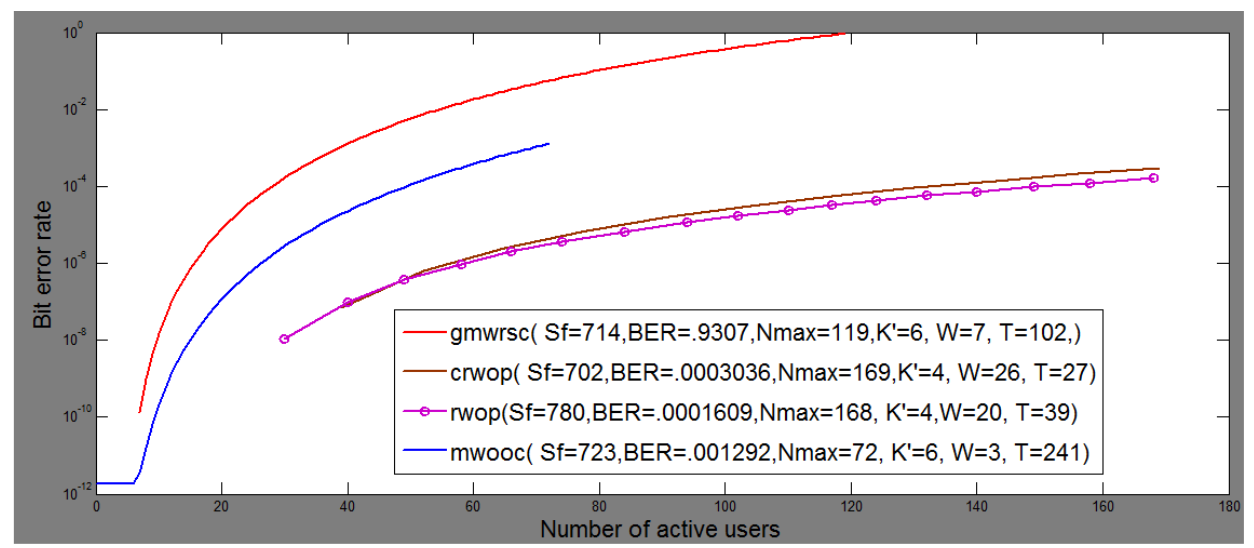

Figure 3. BER vs Number of users with similar spreading factor

Again analysing Table 3 and Figure 3, bit error rate is minimum at number of users 780 which is shown by RWOP. Again RWOP gives high efficiency than other.

Using same technique, again taking the spreading factor is 1430 for GMWRSC, 1482 for C RWOP, 1540 for RWOP, 1505 for MWOOC. For above mentioned spreading factor, different type of parameters such as BER, Nmax, K', WI and T corresponding each code families are generated and suitably placed in Table 4.

Table 2.4 Variation of BER with similar cardinality for four types of code families

\begin{tabular}{lllll}
\hline & GMWRSC & CRWOP & RWOP & MWOOC \\
\hline Sf & 1430 & 1482 & 1540 & 1505 \\
BER & .2734 & .0002595 & .0001313 & .07266 \\
Nmax & 143 & 361 & 334 & 250 \\
$\mathrm{~K}^{\prime}$ & 10 & 4 & 4 & 4 \\
WI & 11 & 38 & 28 & 5 \\
$\mathrm{~T}$ & 130 & 39 & 55 & 301 \\
\hline
\end{tabular}

Using data from Table 4, a graph is plotted between numbers of users in horizontal axis BER along vertical axis, which is shown in Figure 4 Again analysing Table 4 and Figure 4, it is revealed that RWOP code family gives lowest BER corresponding Nmax 334 and spreading factor 1540, however CRWOP gives largest Nmax and less spreading factor with respect to high BER, so it is found that CROWP has more BER then RWOP. 


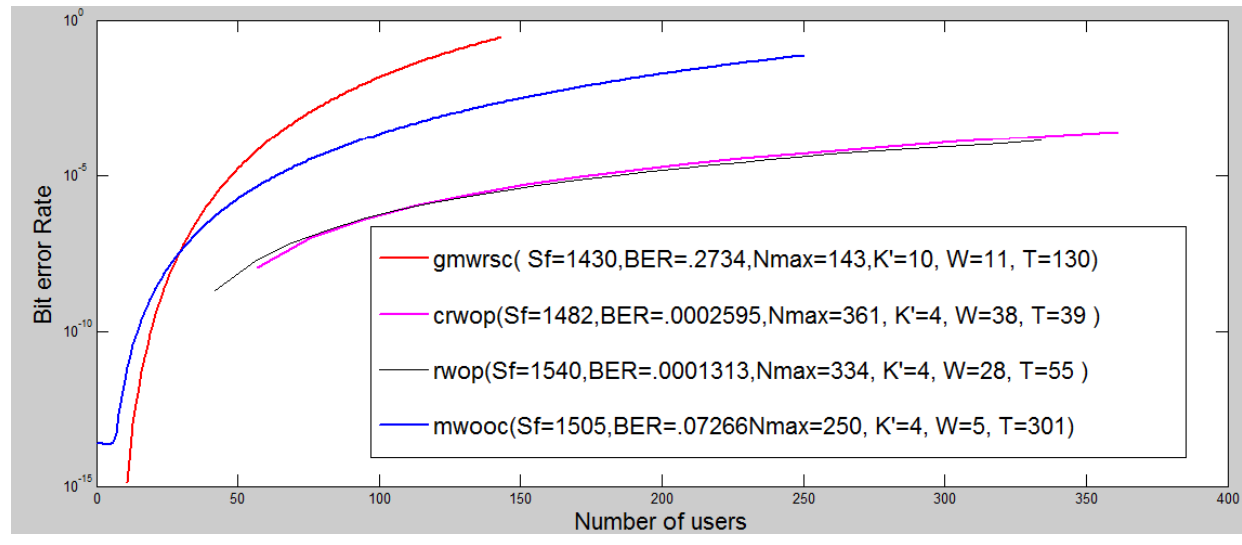

Figure 4. BER vs Number of users with similar spreading factor

It can be seen from Figures 1 and 2 that for similar values of cardinality, the BER is lowest in case of rwop code families. In the same manner, we see that the BER is lowest in rwop code families for similar values of spreading factor from Figures 3 and 4.

In this analysis, we analysed and compared the performance of $2 \mathrm{D}$ code families based on different parameters i.e. cardinality, bit error rate, spreading factor and weight. Existing fiber-optic communication technologies have bit error rates of the order of 10-8. In most cases, rwop code family is better in comparison to other code families. From our discussions, it is observed that the performance of rwop code is better than the other compared code families. In most practical cases, OOCs with $\lambda c=2,3$ perform better than OOCs with $\lambda c=1$, while having a much bigger cardinality due to less number of active users at any given point of time. The OOC is a versatile code which allows huge numbers of asynchronous users for transmitting information to destination. The gmwrsc families have highest cardinality followed by mwooc, crwop and rwop code families for equivalent spreading factor and weight.

We observe that, when $\operatorname{Nmax}=169$ for gmwrsc family, crwop code family, rwop code family and $\mathrm{Nmax}=162$ for mwooc family, gmwrsc family gives highest efficiency for less number of users $(<58)$. But rwop code family gives better performance for more number of users $(>58)$. Similarly, when Nmax $=361$ for gmwrsc family, crwop code family, Nmax=385 for rwop code family and Nmax=375 for mwooc family, it is seen that, again gmwrsc family gives high efficiency for less number of users $(<200)$. But rwop code family gives high efficiency for more number of users $(>200)$. Considering spreading factor it is observed that rwop and crwop code families present high efficiency than others.

\section{CONCLUSION}

A review of the construction and performance for existing FOCDMA code families is undertaken. The performance of four different two dimensional code families spread in the wavelength and time domains is analysed. The variation of bit error rate with number of active users is studied thoroughly for different sets of parameters. The different parameters considered here are cardinality, number of wavelengths, temporal length, spreading factor and weight. The performance analysis for different values of parameters leads to the advantages and disadvantages of each code family. The comparison result revealed, RWOP code family gives high performance with respect to both cardinality. Different types of code family are analysed for investing the performance of FOCDMA. It is found that different parameters such as (Nmax)Bit error rate(BER), Spreading Factor(Sf), Weight(K'),Number of Wavelengths(WI), and number of time chips(T), are played vital role to discuss the same. Finally simulation result revealed that RWOP code family gives high performance then other.

Analysing above concluding remarks, we can say gmwrsc and rwop code families give better performance for similar cardinality. The rwop and crwop code families give better performance for similar spreading factor and weight. The gmwrsc families present highest efficiency for similar bit error rate.

In this section, it is explained the results of research and at the same time is given the comprehensive discussion. Results can be presented in figures, graphs, tables and others that make the reader understand easily [2], [5]. The discussion can be made in several sub-chapters. 


\section{REFERENCES}

[1] A. Stok; E. Sargent, "System performance comparison of optical CDMA and WDMA in a broadcast local area network," Communications Letters, IEEE , vol. 6, no. 9, pp. 409-411, Sep 2002.

[2] G.-C. Yang; W. C. Kwong, "Performance comparison of multiwavelength CDMA and WDMA + CDMA for fiberoptic networks," IEEE Transactions on Communications , vol. 45, no. 11, pp. 1426-1434, November 1997.

[3] W. C. Kwong; G.-C. Yang; V. Baby; C.-S. Br`es; P. R. Prucnal, "Multiple-wavelength optical orthogonal codes under prime-sequence permutations for optical CDMA," IEEE Transactions on Communications, vol. 53, no. 1, pp. 117-123, January 2005.

[4] M. Ravi Kumar; S. S. Pathak; N. B. Chakrabarti , "Design and Performance Analysis of Code Families for MultiDimensional Optical CDMA", IET Communications , vol. 3, no. 8, pp. 1311 - 1320, 2009

[5] M. R. Kumar , "Spreading codes for all-optical code division multiple access communication systems,"Ph.D. dissertation, IIT, Kharagpur, G. S. Sanyal School of Telecommunications, Kharagpur,India, Jul. 2011.

[6] W. Huang; M. Nizam; I. Andonovic; M. Tur, "Coherent optical CDMA (OCDMA) systems used for high-capacity optical fiber networks-system description, OTDMA comparison, and OCDMA/WDMA networking," Lightwave Technology, Journal of , vol.18, no.6, pp.765-778, Jun 2000.

[7] R. Pickholtz; L. Milstein; D. Schilling , "Spread spectrum for mobile communications," Vehicular Technology, IEEE Transactions on , vol.40, no.2, pp.313-322, May 1991.

[8] J. Y. Hui, "Pattern code modulation and optical decoding - a novel codedivision multiplexing technique for multifiber networks," IEEE Journal on Selected Areas in Communications, vol. 3, no. 6, pp. 916-927, November 1985.

[9] W. C. Kwong and P. R. Prucnal , “'synchronous' CDMA demonstration for fiber-optic networks with optical processing," Electronics Letters, vol. 26, no. 24, pp. 1990-1992, November 1990.

[10] W. Kwong; P. Perrier; P. Prucnal, "Performance comparison of asynchronous and synchronous code-division multiple-access techniques for fiber-optic local area networks," Communications, IEEE Transactions on, vol. 39, no. 11 , pp. $1625-1634$, nov 1991.

[11] M. Karbassian; H. Ghafouri-Shiraz, "Performance analysis of heterodyne-detected coherent optical CDMA using a novel prime code family," Lightwave Technology, Journal of, vol. 25, no. 10, pp. 3028-3034, Oct 2007.123

[12] R. Fuji-Hara; Y. Miao, "Optical orthogonal codes: their bounds and new optimal constructions," Information Theory, IEEE Transactions on, vol. 46, no. 7, pp. 2396 -2406, nov 2000.

[13] C.-S. Bres; P. Prucnal, "Code-empowered lightwave networks," Lightwave Technology, Journal of, vol. 25, no. 10, pp. 2911-2921, Oct. 2007.

[14] S. Jindal; N. Gupta, "Performance evaluation of optical CDMA based 3D code with increasing bit rate in local area network," in Computational Technologies in Electrical and Electronics Engineering, 2008. SIBIRCON 2008. IEEE Region 8 International Conference on, July 2008, pp. 386-388.

[15] F. R. K. Chung; J. A. Salehi; V. K. Wei, "Optical orthogonal codes:design, analysis, and applications," IEEE Transactions on Information Theory, vol. 35, no. 3, pp. 595-604, May 1989.

[16] A. A. Shaar; P. A. Davies, "Prime sequences: quasi-optimal sequences for OR channel code division multiplexing," Electronics Letters, vol. 19, no. 21, pp. 888-890, October 1983.

[17] W. C. Kwong; G.-C. Yang; J.-G. Zhang, "2n prime-sequence codes and coding architecture for optical codedivision multiple-access," IEEE Transactions on Communications, vol. 44, no. 9, pp. 1152-1162, September 1996.

[18] J. A. Salehi, "Code division multiple-access techniques in optical fiber networks-part I: fundamental principles," IEEE Transactions on Communications, vol. 37, no. 8, pp. 824-833, August 1989.

[19] R. A. Brualdi , Introductory Combinatorics, 1st ed. North-Holland, New York: North-Holland, 1977.

[20] C. Argon; R. Erg"ul, "Optical CDMA via shortened optical orthogonal codes based on extended sets," Optics Communications, vol. 116, no. 4-6, pp. 326-330, February 1995.

[21] C. Argon , "Systematic optical orthogonal code design techniques use extendedsets," in IEEE International Conference on Communications, IEEE.Istanbul, Turkey: IEEE, June 2006.

[22] K. Fouli; M. Maier, "OCDMA and optical coding: Principles, applications, and challenges [topics in optical communications]," CommunicationsMagazine, IEEE, vol. 45, no. 8, pp. 27-34, August 2007.

[23] B. Ghaffari; M. Matinfar; J. Salehi, "Wireless optical CDMA LAN:digital design concepts," Communications, IEEE Transactions on, vol. 56,no. 12, pp. 2145-2155, December 2008.

[24] C.-C. Yang; J.-F. Huang; Y.-H. Wang, "Multipulse-per-row codes for high-speed optical wavelength/time CDMA networks," Photonics Technology Letters, IEEE, vol. 19, no. 21, pp. 1756-1758, Nov.1, 2007.

[25] S. Kim; K. Yu; N. Park, "A new family of space/wavelength/time spread three-dimensional optical code for OCDMA networks," Lightwave Technology, Journal of, vol. 18, no. 4, pp. 502-511, Apr 2000. 